\title{
Simulation of different options of the Inner Tracker for Novosibirsk Super Charm-Tau Factory Detector
}

\author{
T.V. Maltsev ${ }^{1,2, *}$, L.I. Shekhtman ${ }^{1,2}$, A.V. Sokolov ${ }^{1,2}$ and V.K. Vadakeppattu ${ }^{2}$ \\ ${ }^{1}$ Budker Institute of Nuclear Physics, Novosibirsk, Russia \\ ${ }^{2}$ Novosibirsk State University, Novosibirsk, Russia
}

\begin{abstract}
Inner Tracker of Novosibirsk Super Charm-Tau Factory Detector has to measure momenta of soft hadrons, which do not reach the drift chamber; complement the drift chamber in measuring the momenta; detect secondary vertices of short-lived particles. Thus, proper choice of the option for the Inner Tracker is of significant interest. The simulation of charged pions propagation in the perpendicular direction to the beam axis was carried out with DD4HEP program based on GEANT4. Three options were considered: 4-layered Silicon microstrip detector, 4-layered cylindrical Gas Electron Multiplier (GEM) detector and Time Projection Chamber (TPC). The simulated Detector was located in $1.5 \mathrm{~T}$ magnetic field directed along beam axis. The simulation shows that pions with initial momenta less than $50 \mathrm{MeV} / \mathrm{c}$ do not pass through the beampipe and cannot be registered. Pions with momenta above $65 \mathrm{MeV} / \mathrm{c}$ leave energy depositions in all 4 layers of the Inner Tracker based on Silicon, and their trajectories can be reconstructed. Cylindrical GEM detector provides reconstruction possibility (hits in 4 layers) for pions with momenta more than $60 \mathrm{MeV} / \mathrm{c}$. TPC with thin inner wall provides reconstruction of pions with momenta higher than $55 \mathrm{MeV} / \mathrm{c}$. However, in this case the reconstruction procedure will be much more complicated due to a large number of background particle tracks in the sensitive volume.
\end{abstract}

\section{Introduction}

Physics program of experiments at the Novosibirsk Super Charm-Tau Factory Detector [1] with a peak luminosity of $10^{35} \mathrm{~cm}^{-2} \mathrm{~s}^{-1}$ in the energy region $2 E=2-7 \mathrm{GeV}$ is dedicated to studies of rare decays of $D$ mesons, $\tau$ lepton, $D^{0} \overline{D^{0}}$ oscillations and searches for so-far unobserved lepton-flavor-violating $\tau$ decays, in particular, $\tau \rightarrow \mu \gamma$ decay. The proposed program requires the development of a universal magnetic detector (Fig. 1). Inner Tracker is placed between the vacuum chamber and the drift chamber. It provides the detection solid angle up to $98 \%$. Inner Tracker is a cylinder with a length of $60 \mathrm{~cm}$, inner diameter is $3 \mathrm{~cm}$, outer diameter is $40 \mathrm{~cm}$. Its task is to detect secondary vertices from the decays of short-lived particles, such as $K_{S}$ or $\Lambda$. Furthermore Inner Tracker complements the drift chamber in measuring the momenta of charged particles. Thus, the reconstruction of soft hadrons is the primary task of Inner Tracker.

\footnotetext{
*e-mail: T.V.Maltsev@inp.nsk.su
} 


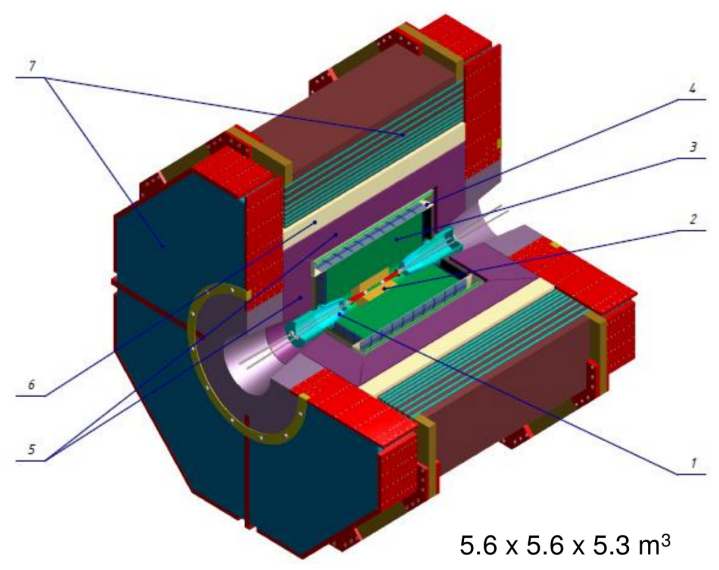

1. Vacuum pipe

2. Inner tracker

3. Drift chamber

4. PID

5. Calorimeter

6. SC magnet

7. Muon system

Figure 1. Novosibirsk Super Charm-Tau Factory Detector.

The simulated distribution of $\pi+$ mesons (resulting from the reaction $e^{+} e^{-} \rightarrow D D^{*}$ ) on their momentum is presented in Fig. 2 [2]. The determination of minimum momentum, starting from which the pion reconstruction becomes possible with Inner Tracker, was the subject of current simulation. The simulation was carried out with DD4HEP [3] and GEANT4 [4] programs. Pions with different momenta were directed perpendicular to the Inner Tracker axis. Magnetic field of $1.5 T$ was directed along the beam axis. Beampipe (vacuum pipe) in the simulation consists of $3.0 \mathrm{~mm}$ of Beryllium and $0.5 \mathrm{~mm}$ of Paraffin. Paraffin is located in the middle of two Beryllium layers, each of which is $1.5 \mathrm{~mm}$ width. The simulation was carried out for three options of Inner Tracker:

1. Four-layered cylindrical Silicon microstrip detector (Si-strips);

2. For-layered cylindrical GEM detector [5], [6];

3. Time Projection Chamber (TPC).

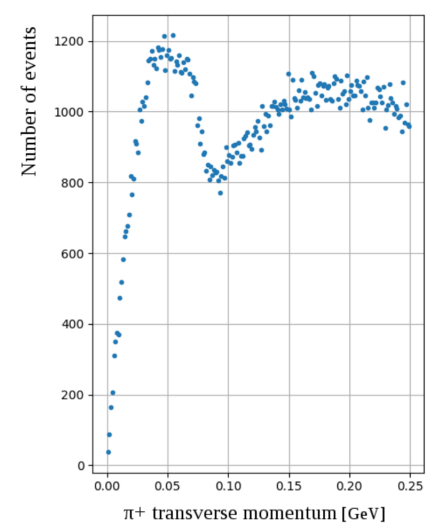

Figure 2. Distribution of $\pi+$ mesons (resulting from the reaction $e^{+} e^{-} \rightarrow D D^{*}$ ) on their transverse momentum [2]. 


\section{Four-layered Silicon-strips detector}

First option of the Inner Tracker is based on 4 layers of $0.32 \mathrm{~mm}$ Silicon and $0.4 \mathrm{~mm}$ Carbon fiber (Fig. 3). Air at the standard conditions is located outside the Silicon and Carbon layers.
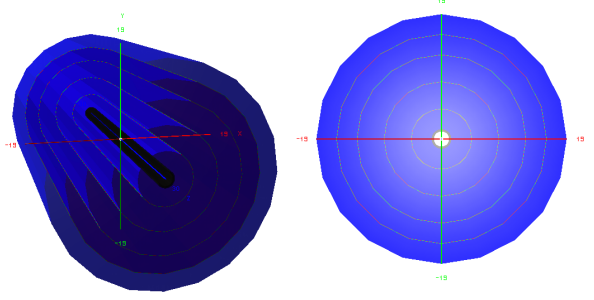

Figure 3. Silicon-strips Inner Tracker, described in the DD4HEP simulation.

The simulation shows that pions with momenta less than $50 \mathrm{MeV} / \mathrm{c}$ do not pass through the beampipe. Starting from $p_{\pi}=55 \mathrm{MeV} / \mathrm{c}$ the first layer is reached by pions. Pions with momenta $60 \mathrm{MeV} / \mathrm{c}$ pass through two layers. Starting from $p_{\pi}=65 \mathrm{MeV} / \mathrm{c}$ all 4 layers are reached by pions. Pions return back with momenta around $70 \mathrm{MeV} / \mathrm{c}$. Pions with $p_{\pi}=$ $75 \mathrm{MeV} / \mathrm{c}$ cross layers twice. Examples of simulation results are shown in Fig. 4. The reconstruction of pions with hits in 4 layers for Si-strips option of Inner Tracker is possible for pions with momenta, greater than $65 \mathrm{MeV} / \mathrm{c}$.
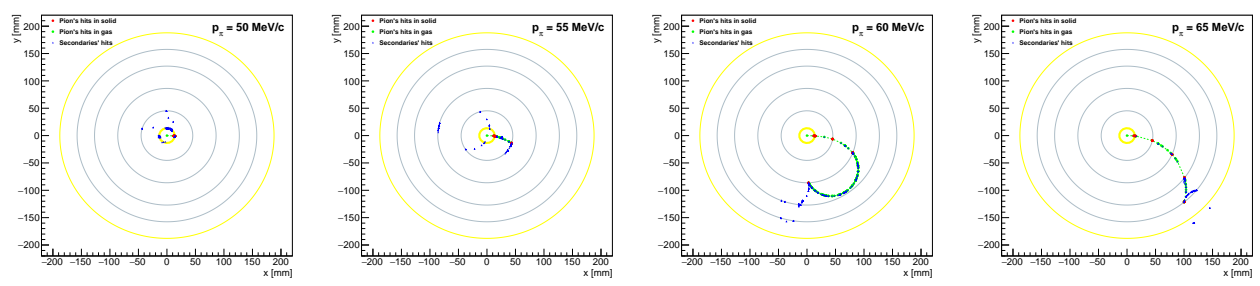

Figure 4. Cross-section of Inner Tracker based on Si-strips with hits, left by pion and secondary particles.

\section{Four-layered GEM detector}

Second option of the Inner Tracker is based on 4 layers of triple GEM detectors (Fig. 5). First layer of each GEM detector is Kapton $50 \mu \mathrm{m}$ width. Then a Copper $5 \mu \mathrm{m}$ width (drift electrode) is placed. Drift gap is $3 \mathrm{~mm}$ width. Two transport and induction gaps are equally $1.5 \mathrm{~mm}$ width. Gas volumes inside GEM detectors are filled with gas $\operatorname{Ar}(75 \%)-\mathrm{CO}_{2}(25 \%)$. Each GEM consists of $50 \mu \mathrm{m}$ Kapton, covered on both sides with $5 \mu \mathrm{m}$ Copper, besides that, GEM holes are accounted by decreasing density in Copper and Kapton (factor 0.8). Last layers in each GEM detector are anode (Copper $5 \mu \mathrm{m}$ width) and Kapton $50 \mu \mathrm{m}$ width. Air is placed outside GEM detectors. 


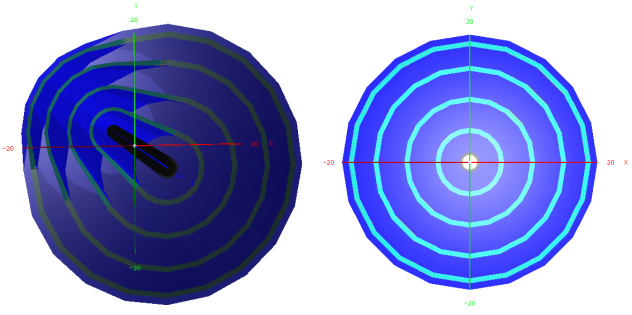

Figure 5. Cylindrical GEM Inner Tracker, described in the DD4HEP simulation.

Starting from $p_{\pi}=55 \mathrm{MeV} / \mathrm{c}$ two GEM layers can be reached by pions. Pions with momenta $60 \mathrm{MeV} / \mathrm{c}$ pass through three layers and curve back. Pions with momenta slightly greater than $60 \mathrm{MeV} / \mathrm{c}$ leave hits in $4 \mathrm{GEM}$ layers. Starting from $p_{\pi}=65 \mathrm{MeV} / \mathrm{c}$ all 4 layers are crossed by pions minimum twice. Pions can be registered precisely for momenta, greater than $70 \mathrm{MeV} / \mathrm{c}$ in case of their transverse motion. Examples of simulation results are shown in Fig. 6. The reconstruction of pions with hits in 4 layers for CGEM option of Inner Tracker is possible for pions with momenta, greater than $60 \mathrm{MeV} / \mathrm{c}$.
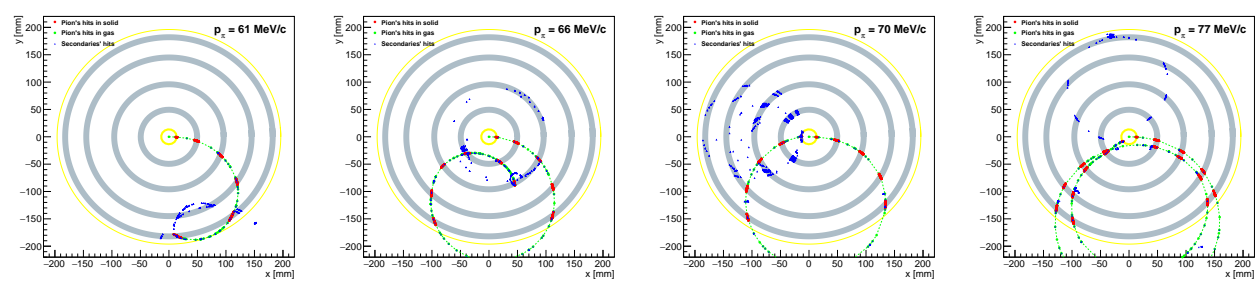

Figure 6. Cross-section of Inner Tracker based on cylindrical GEM detectors with hits, left by pion and secondary particles.

\section{Time Projection Chamber}

The last considered option - TPC (Fig. 7) has inner and outer walls, which restrict the gas volume, filled with gas mixture $\mathrm{Ar}(80 \%)-\mathrm{CO}_{2}(20 \%)$ at atmospheric pressure. Two suboptions of TPC were simulated:

1. TPC with standard outer and inner walls, each of which has the same width and consists of $1 \mathrm{~mm} \mathrm{G} 10+0.1 \mathrm{~mm}$ Teflon $+15 \mu \mathrm{m}$ Copper;

2. TPC with standard outer wall and thin inner wall, where thin inner wall consists of $50 \mu m$ Kapton $+0.1 \mathrm{~mm}$ Teflon $+5 \mu m$ Copper.

Pions with momenta greater than $55 \mathrm{MeV} / \mathrm{c}$ pass through thin inner wall but they do not pass through standard inner wall. Pions with momenta greater than $60 \mathrm{MeV} / \mathrm{c}$ can be reconstructed for both TPC sub-options. Outer wall also influence the pions path in their further motion. Pions with momenta, higher than $70 \mathrm{MeV} / \mathrm{c}$ reach the outer wall minimum thrice. Soft electrons leave a lot of ionization in the volume. Examples of simulation results are shown in Fig. 8. The reconstruction of pions for TPC option of Inner Tracker is possible:

1. For standard wall sub-option $-p_{\pi}>60 \mathrm{MeV} / \mathrm{c}$;

2. For thin wall sub-option $-p_{\pi}>55 \mathrm{MeV} / \mathrm{c}$. 


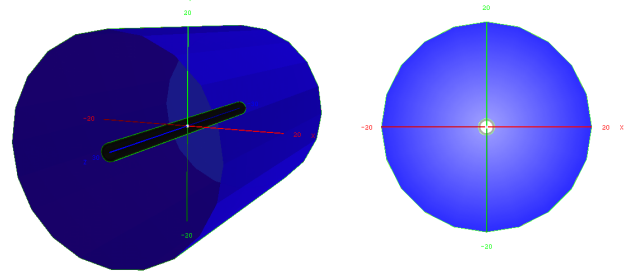

Figure 7. TPC Inner Tracker, described in the DD4HEP simulation.
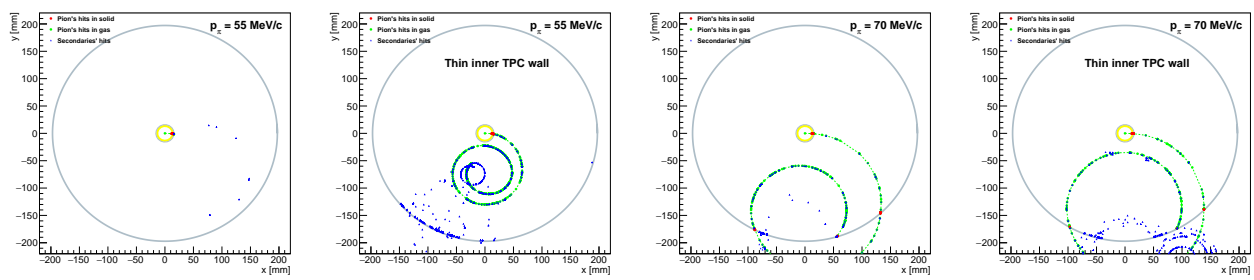

Figure 8. Cross-section of Inner Tracker based on TPC with hits, left by pion and secondary particles.

\section{Conclusions}

Different options of the Inner Tracker for Novosibirsk Super Charm-Tau Factory Detector were considered in order to choose the best one for soft hadrons reconstruction. The simulation of charged pions propagation in the perpendicular direction to the beam axis, carried out with DD4HEP and GEANT4, was done for three options: 4-layer Silicon-strips detector, 4-layer cylindrical GEM detector and Time Projection Chamber (TPC). The simulation results demonstrate that pions with initial momenta less than $50 \mathrm{MeV} / \mathrm{c}$ do not pass through the beampipe. Pions with momenta above $65 \mathrm{MeV} / \mathrm{c}$ provide energy depositions in all 4 layers of the Inner Tracker based on Silicon microstrip detector, hence their trajectories can be reconstructed. Cylindrical GEM detector provides reconstruction possibility (hits in 4 layers) for pions with momenta greater than $60 \mathrm{MeV} / \mathrm{c}$. TPC with thin inner wall provides reconstruction of pions with momenta higher than $55 \mathrm{MeV} / \mathrm{c}$. But in this case the reconstruction procedure is assumed to be more complicated due to large number of background particle tracks.

\section{References}

[1] Super Charm-Tau Factory, https://ctd.inp.nsk.su/c-tau/.

[2] Private communication with V. S. Vorobiev.

[3] DD4HEP, https://dd4hep.web.cern.ch/dd4hep/.

[4] GEANT4 collaboration, S. Agostinelli et al., GEANT4: a simulation toolkit, Nucl. Instrum. Meth. A 506 (2003) 250.

[5] F. Sauli, GEM: a new concept for electron amplification in gas detectors, Nucl. Instrum. Methods A 386 (1997) 531.

[6] A. Balla et al., The KLOE-2 Inner Tracker: Detector commissioning and operation, Nucl. Instrum. Methods A 845 (2017) 266. 